\title{
Ischaemic aetiology predicts exercise dyssynchrony in patients with heart failure with reduced ejection fraction
}

\author{
Jakub Stępniewski, Grzegorz Kopeć, Wojciech Magoń, Piotr Podolec \\ Department of Cardiac and Vascular Diseases, Jagiellonian University Medical College, John Paul Il Hospital, Krakow, Poland
}

\begin{abstract}
A bstract
Background: Left ventricular (LV) dyssynchrony is common in patients with heart failure with reduced ejection fraction (HFrEF). However, various conditions, including exercise, may affect its presence. LV dyssynchrony at exercise (ExDYS) has been associated with lower cardiac performance and exercise capacity but with higher cardiac resynchronisation therapy (CRT) response. Therefore, understanding the mechanisms underlying ExDYS may improve patient selection for CRT.

Aim: We sought to identify predictors of ExDYS among patients with HFrEF and prolonged QRS duration.

Methods: Consecutive patients with stable chronic heart failure, LV ejection fraction (LVEF) $<35 \%$, sinus rhythm, and QRS $\geq 120$ ms were eligible. Two-dimensional echocardiography and tissue-Doppler were performed at rest and during peak cyclo-ergometer exercise to assess LV systolic (LVEF) and diastolic functions (mitral E-to-e'-wave velocities [E/e']) and dyssynchrony. Dyssynchrony was defined as a maximal difference between time-to-peak systolic velocities of $\geq 65 \mathrm{~ms}$ from opposing basal segments.

Results: We included 48 patients (aged $63.7 \pm 12.2$ years, $81.3 \%$ male). Ischaemic aetiology (ischaemic cardiomyopathy [ICM]) was present in $23(47.9 \%)$ patients. Dyssynchrony at rest (rDYS) was present in $32(66.6 \%)$ patients, while ExDYS was seen in 23 (47.9\%). ExDYS correlated with ICM, lower LVEF, and higher E/e' ratio. ICM remained a significant predictor of ExDYS in multiple regression model (odds ratio [OR] 4.3, 95\% confidence interval $[\mathrm{Cl}] 1.2-15.7, p=003$ ). On exercise, 19 (39.5\%) patients changed the rDYS status. Although exercise-induced dyssynchronisation was observed only in ICM patients, exercise-induced resynchronisation was more likely in patients with lower rest $\mathrm{E} / \mathrm{e}^{\prime}$ ratio (OR 0.85, 95\% $\left.\mathrm{Cl} 0.75-0.97, \mathrm{p}=0.02\right)$.

Conclusions: Ischaemic aetiology of HFrEF is an important predictor of ExDYS. Restoration of LV synchronicity during exercise is more likely in patients with less advanced LV diastolic dysfunction.

Key words: stress echocardiography, dilated cardiomyopathy, QRS prolongation, cardiac resynchronisation therapy, left bundle branch block
\end{abstract}

Kardiol Pol 2018; 76, 10: 1450-1457

\section{INTRODUCTION}

In most recent studies, the presence of left ventricular (LV) dyssynchrony at exercise was shown to predict lower cardiac performance and exercise capacity in patients with heart failure with reduced ejection fraction (HFrEF) [1, 2]. Interestingly, patients with exercise dyssynchrony were also more likely to respond to cardiac resynchronisation therapy (CRT) [3]. Therefore, understanding the mechanisms behind exercise-related dyssynchrony may improve patient selection for CRT.

In the present study, we aimed to identify predictors of exercise dyssynchrony among patients with HFrEF and prolonged QRS duration.

\section{METHODS \\ Study population}

We prospectively enrolled consecutive HF patients who were considered for CRT device implantation at the John Paul II Hospital in Krakow, Poland in 2013 and 2014. Inclusion criteria comprised the following: (1) New York Heart Association (NYHA) functional class II-IV despite optimal medical therapy and optimal coronary revascularisation; (2) LV ejection fraction (LVEF) $\leq 35 \%$ and QRS duration $\geq 120 \mathrm{~ms}$ on 12-lead electrocardiogram; and (3) sinus rhythm and no exacerbations within the past three months. Heart failure (HF) of ischaemic aetiology (ischaemic cardiomyopathy [ICM]) was diagnosed 
if a patient had a history of myocardial infarction, coronary revascularisation, or had angiographically significant stenotic lesions of $>50 \%$ in coronary arteries, and a diagnosis of non-ischaemic HF (dilated cardiomyopathy [DCM]) was made when there was no history of coronary artery disease. Patients with a history of any cardiac implantable electronic devices, persistent atrial fibrillation, or significant respiratory, neurological, or orthopaedic disorder precluding cycle ergometer exercise were excluded from the study.

All patients provided their written, informed consent to participate in this study. The study was performed in accordance with the Declaration of Helsinki and was approved by the Institutional Ethical Committee at the Jagiellonian University in Krakow, Poland (KBET/110/B/2013).

All measurements and patients' medical records were prospectively obtained by the authors themselves.

\section{Echocardiography}

All echocardiographic examinations were performed with the use of a commercially available Vivid 7 device (GE Medical System, Horten, Norway) equipped with a phased array 3.5-MHz transducer and tissue Doppler imaging (TDI) software. The images were stored digitally for offline analysis on EchoPac software (GE Vingmed, Horten, Norway). Conventional M-mode, two-dimensional (2D), and Doppler parameters were calculated. TDI data were recorded in apical four-, two-, and three-chamber views with sector size and depth optimisation for the highest frame rate. Regional time-velocity curves were produced offline from the stored TDI colour images by placing sample volumes over six basal LV segments. All measurements were performed by an experienced echocardiographer by averaging three or more consecutive heart beats.

Left ventricular volumes and LVEF, as a measure of LV systolic function, were calculated with 2D-echocardiography from apical four- and two-chamber views, using the biplane disc summation method [4], and similarly for left atrial volume (LAV). Chamber volumes were normalised for body surface area.

Calculation of the $\mathrm{E} / \mathrm{e}^{\prime}$ ratio, adopted as a measure of LV diastolic function, was performed by dividing peak mitral E-wave velocity acquired with pulsed-wave Doppler by TDI-derived pulsed-wave, mean septal, and lateral mitral annulus early diastolic velocities ( $\left.\mathrm{e}^{\prime}\right)$.

A conventional 16-segment LV model was used to characterise regional contractility of the LV walls by scoring each segment from one to four on the basis of systolic thickening and motion [4]. Wall motion score index (WMSI) was calculated as the sum of all scores divided by the number of segments visualised. Contractile reserve (CR) was defined as a decrease of WMSI by at least 0.20 from rest to peak exercise.

The severity of mitral regurgitation (MR) was evaluated qualitatively and graded from I (mild) to IV (severe).

\section{Exercise stress echocardiography}

Following the examination at rest, exercise echocardiography was performed on cycle ergometer (Ergoline 9000 Ergoline $\mathrm{GmbH}$, Bitz, Germany) in semi-recumbent position with increasing workload. The initial workload was $20 \mathrm{~W}$ and was raised every $2 \mathrm{~min}$ by additional $20 \mathrm{~W}$. The tests were terminated on patient's request after achieving maximal effort. Echocardiographic recordings were done continuously throughout the exercise and stored digitally for subsequent off-line analysis on EchoPAC GE medical software. Peak exercise measurements were obtained from the final 2-min cycle. Each measurement was derived from at least three heart beats and expressed as the mean. No changes to patients' pharmacotherapy were made for the sole purpose of the stress test.

\section{Dyssynchrony evaluation}

Time-velocity curves of six basal segments were used to assess LV dyssynchrony at rest (rDYS) and at peak exercise (ExDYS). Time-to-peak systolic velocity (Ts) was measured from the onset of the QRS complex to the peak myocardial systolic velocity in each of the six basal segments. The differences between Ts of opposing wall segments were calculated to determine opposing wall delays. At least one opposing wall delay $(\operatorname{maxTsD}) \geq 65 \mathrm{~ms}$ was indicative of rDYS and ExDYS. Ts was corrected for the RR interval using the Bazett formula.

\section{Statistical analysis}

Categorical variables were described as counts and percentages, and continuous variables as means \pm standard deviation or median and interquartile range. We used the unpaired Student t test for normally distributed variables, the Mann-Whitney $U$ test for non-normally distributed continuous data, and the $\chi^{2}$ test for categorical data to compare patients with and without ExDYS. In order to test the significance level of rest and peak exercise differences, we used the paired Student $t$ test and the Wilcoxon test.

We used univariate logistic regression analysis to evaluate the association between the presence of ExDYS and its potential predictors, including age, sex $(0-$ male, 1 - female), HF aetiology (0 - DCM, 1 - ICM), QRS duration and morphology $(0-$ non-specific intraventricular conduction delay [IVCD], 1 — left bundle branch block [LBBB]), LV end-diastolic volume index (LVEDV index $)$, markers of LV systolic and diastolic functions (LVEF and E/e' ratio, respectively), and the presence of rDYS. Similarly, using univariate logistic regression models we investigated the association between exercise-induced changes of rDYS status, including exercise-induced resynchronisation, and exercise-induced dyssynchronisation and its potential predictors including age, sex $(0-$ male, 1 - female), HF aetiology ( 0 - DCM, 1 - ICM), QRS duration and morphology $\left(0-\right.$ IVCD $_{1} 1$ - LBBB $)$, LVEDV $_{\text {index' }}$ 
Table 1. Clinical characteristics of the studied patients

\begin{tabular}{|c|c|c|c|c|}
\hline Variable & All patients $(n=48)$ & Without ExDYS $(n=25)$ & With ExDYS $(n=23)$ & p \\
\hline Age [years] & $63.7 \pm 12.2$ & $64 \pm 11.7$ & $63.3 \pm 12.9$ & 0.85 \\
\hline Women/men & $9(18.7) / 39(81.3)$ & $6(24) / 19(76)$ & $3(13) / 20(87)$ & 0.55 \\
\hline BMI [kg/m²] & $26.5 \pm 3.8$ & $26.6 \pm 4.3$ & $26.3 \pm 3.3$ & 0.78 \\
\hline Ischaemic/non-ischaemic HF & $23(47.9) / 25(52.1)$ & $8(32) / 17(68)$ & $15(65.2) / 8(34.8)$ & 0.04 \\
\hline NYHA class: & & & & 0.43 \\
\hline$\|$ & $12(25)$ & $6(24)$ & $6(26.1)$ & \\
\hline III & $30(62.5)$ & $18(72)$ & $12(52.2)$ & \\
\hline IV & $6(12.5)$ & $1(4)$ & $5(21.7)$ & \\
\hline NT-proBNP [pg/mL] & $1667(503-3309)$ & $1460(628-2616)$ & $2009(491-5306)$ & 0.22 \\
\hline HR [bpm] & $70.6 \pm 8.9$ & $71.1 \pm 9.4$ & $70.1 \pm 8.5$ & 0.7 \\
\hline QRS duration [ms]: & $150(120-160)$ & $140(120-160)$ & $160(122.5-160)$ & 0.32 \\
\hline $120-129 \mathrm{~ms}$ & $13(27)$ & $8(61)$ & $5(39)$ & \\
\hline $130-149 \mathrm{~ms}$ & $11(23)$ & $6(55)$ & $5(45)$ & \\
\hline$\geq 150 \mathrm{~ms}$ & $24(50)$ & $11(46)$ & $13(54)$ & \\
\hline LBBB/IVCD & $28(58.3) / 20(41.7)$ & $13(52) / 12(48)$ & $15(65.2) / 8(34.8)$ & 0.52 \\
\hline$\beta$-blocker & $47(97.9)$ & $24(96)$ & $23(100)$ & 0.96 \\
\hline$A C E I$ or $A R B$ & $47(97.9)$ & $24(96)$ & $23(100)$ & 0.96 \\
\hline ARA & $44(91.6)$ & $22(88)$ & $22(95.6)$ & 0.66 \\
\hline Loop diuretics & $44(91.6)$ & $23(92)$ & $21(91.3)$ & 0.66 \\
\hline
\end{tabular}

Data are shown as number (percentage), mean \pm standard deviation, or median and interquartile range. ACEI — angiotensin converting enzyme inhibitor; ARA — aldosterone receptor antagonist; ARB — angiotensin receptor blocker; BMI — body mass index; HF — heart failure; HR — heart rate; IVCD — non-specific intraventricular conduction disturbances; LBBB — left bundle branch block; NT-proBNP — N-terminal pro-B-type natriuretic peptide; NYHA — New York Heart Association

and markers of LV systolic and diastolic functions (LVEF and $\mathrm{E} / \mathrm{e}^{\prime}$ ratio, respectively). Multiple stepwise logistic regression analysis was used to evaluate the associations between the presence of ExDYS or exercise-induced changes of rDYS status and their potential predictors. In these models we used only those potential predictors which were significantly associated with ExDYS or exercise-induced changes of rDYS status in univariate models.

The significance level was set at $p$-value $<0.05$. Statistical analyses were performed with Statistica PL software (version 12, StatSoft, Inc., Krakow, Poland) and MedCalc version 11.6.1.0 (MedCalc Software, Mariakerke, Belgium).

\section{RESULTS}

\section{Patients' characteristics}

There were 54 patients eligible for the study, of whom six were excluded due to insufficient quality of echocardiographic recordings. Among 48 enrolled patients aged $63.7 \pm 12.2$ years, men represented the majority $(39 ; 81.3 \%)$. ICM was present in 23 (47.9\%) patients, and DCM in 25 (52.1\%). Thirty (62.5\%) patients were in NYHA class III, $12(25 \%)$ in class II, and six $(12.5 \%)$ in class IV. Median N-terminal pro-B-type natriuretic peptide level was 1667 pg/mL (503-3309 pg/mL). Median
QRS duration was $150 \mathrm{~ms}$ (120-160 ms) with the LBBB morphology present in 28 (58.3\%) patients and non-specific intraventricular conduction disturbances found in 20 (41.7\%). Patients were treated in accordance with contemporary guidelines [5]. Forty-seven (97.9\%) of them used $\beta$-blockers and angiotensin converting enzyme inhibitor or angiotensin receptor blocker and 44 (91.6\%) used aldosterone receptor antagonist. Loop diuretics were used in $44(91.6 \%)$ patients. Clinical characteristics of the study group are presented in Table 1.

\section{Rest and stress echocardiography}

Rest and stress echocardiographic examinations were completed successfully, with no significant adverse events. Stress examinations were terminated at a mean workload of $76.2 \pm 30.5$ W. Detailed rest and exercise echocardiographic parameters are presented in Table 2. Left heart chambers were enlarged with a median LVEDV ${ }_{\text {index }}$ of $169 \mathrm{~mL} / \mathrm{m}^{2}(131-$ $-194 \mathrm{~mL} / \mathrm{m}^{2}$ ) and a mean $\mathrm{LAV}_{\text {index }}$ of $66.7 \pm 25.9 \mathrm{~mL} / \mathrm{m}^{2}$. Global and regional LV systolic functions were decreased with a mean LVEF of $23.6 \% \pm 6 \%$ and a mean WMSI of $2.18 \pm 0.38$. Diastolic $\mathrm{LV}$ function was impaired with mean $\mathrm{E} / \mathrm{e}^{\prime}$ ratio of $17.1 \pm 8$.

Exercise resulted in a decrease in mean $\mathrm{E} / \mathrm{e}^{\prime}$ ratio as compared to rest values $(14.7 \pm 6.2, p=0.03)$. In contrast, 
Table 2. Echocardiographic parameters at rest and at peak exercise

\begin{tabular}{|c|c|c|c|c|}
\hline Variable & All patients $(n=48)$ & Without ExDYS $(n=25)$ & With ExDYS ( $n=23$ ) & p \\
\hline \multicolumn{5}{|l|}{ At rest: } \\
\hline $\operatorname{LVEDV}_{\text {index }}\left[\mathrm{mL} / \mathrm{m}^{2}\right]$ & $169(131-194)$ & $155(122-189)$ & $172(141-204)$ & 0.2 \\
\hline$L A V_{\text {index }}\left[\mathrm{mL} / \mathrm{m}^{2}\right]$ & $66.7 \pm 25.9$ & $57.2 \pm 19.6$ & $76.7 \pm 28.4$ & 0.01 \\
\hline LVEF [\%] & $23.6 \pm 6.0$ & $25.6 \pm 5.7$ & $21.4 \pm 5.7$ & 0.01 \\
\hline E/e' ratio & $17.1 \pm 8.1^{\#}$ & $14.4 \pm 5.6$ & $20 \pm 9.5$ & 0.02 \\
\hline MR [I-IV] & $1(1-2)$ & $1(0.75-2)$ & $2(1-3)$ & 0.02 \\
\hline WMSI & $2.18 \pm 0.38^{\#}$ & $2.1 \pm 0.46^{\#}$ & $2.3 \pm 0.23^{\#}$ & 0.06 \\
\hline Max opposing wall delay [ms] & $85.4 \pm 41.2$ & $74.2 \pm 40^{\#}$ & $97.5 \pm 40^{\#}$ & 0.04 \\
\hline rDYS & $32(66.6)$ & $14(56)$ & $18(78.3)$ & 0.18 \\
\hline \multicolumn{5}{|l|}{ At peak exercise: } \\
\hline Max HR [bpm] & $115.4 \pm 22.1$ & $116.1 \pm 22.2$ & $114.6 \pm 22.5$ & 0.81 \\
\hline Max workload [W] & $80(60-100)$ & $80(60-100)$ & $60(60-100)$ & 0.37 \\
\hline LVEF [\%] & $24.4 \pm 7.0$ & $26.2 \pm 6.6$ & $22 \pm 7.2$ & 0.04 \\
\hline E/e' ratio & $14.7 \pm 6.2^{\#}$ & $13.5 \pm 6.2$ & $16.1 \pm 6$ & 0.16 \\
\hline MR [I-IV] & $1(1-2)$ & $1(1-2)$ & $2(1-3)$ & 0.007 \\
\hline WMSI & $2.07 \pm 0.36^{\#}$ & $1.9 \pm 0.4^{\#}$ & $2.1 \pm 0.2^{\#}$ & 0.11 \\
\hline$C R$ & $16(33.3)$ & $8(32)$ & $8(34.8)$ & 0.91 \\
\hline Max opposing wall delay [ms] & $76.4 \pm 42.8$ & $40.6 \pm 15.6^{\#}$ & $115.2 \pm 24.9^{\#}$ & $<0.03$ \\
\hline
\end{tabular}

Data are shown as number (percentage), mean \pm standard deviation, or median and interquartile range. \#p-value $<0.05$ for rest to peak exercise comparisons; CR - contractile reserve; E/e' ratio - early diastolic mitral velocity to mean early diastolic velocity of the mitral annulus ratio; $\mathrm{LAV}_{\text {inex }}$ - left atrial volume normalised by body surface area; LVEDV ${ }_{\text {index }}$ left ventricular end-diastolic volume normalised by body surface area; $\mathrm{LVEF}$ - left ventricular ejection fraction; MR - mitral regurgitation; rDYS — dyssynchrony at rest; WMSI — wall motion score index

mean $\operatorname{LVEF}(24.4 \% \pm 7.0 \%, \mathrm{p}=0.23)$ remained without significant changes. Mean peak exercise WMSI was lower as compared to the value at rest $(2.07 \pm 0.36, p<0.001)$ and reduced by at least 0.20 at peak exercise in 16 (33.3\%) patients, revealing CR.

\section{Dyssynchrony}

The mean value of maxTsD was $85.4 \pm 41.2 \mathrm{~ms}$ at rest and $76.4 \pm 42.8 \mathrm{~ms}$ at peak exercise $(\mathrm{p}=0.15)$. rDYS was identified in $32(66.6 \%)$ patients, and ExDYS in 23 (47.9\%). Two-thirds (15 [65.2\%]) of patients with ExDYS had ICM as compared to patients without ExDYS, in whom the majority had DCM (17 [68\%]; $p=0.04$; Table 1). Patients with ExDYS as compared to patients without ExDYS had lower mean LVEF and higher mean E/e' ratio (Table 2). No differences in the presence of rDYS were observed between those with or without ExDYS (18 [78.3\%] vs. 14 [56\%], $p=0.18$ ). The WMSI was similar in both groups $(2.3 \pm 0.23$ vs. $2.1 \pm 0.46$, $\mathrm{p}=0.06$, with and without ExDYS, respectively) and it increased in a similar way during exercise in patients with and without ExDYS, irrespective of HF aetiology. The CR was equally prevalent in patients with and without ExDYS (8 [34.7\%] vs. 8 [32\%], p = 0.91).

Univariate logistic regression analysis showed that the presence of ExDYS correlated with ICM (odds ratio [OR] 4;
95\% confidence interval $[\mathrm{Cl}] 1.2-13.2 ; \mathrm{p}=0.02)$, lower LVEF (OR 0.88; 95\% Cl 0.78-0.98; $\mathrm{p}=0.02$ ), and higher E/e' ratio (OR 1.11; 95\% Cl 1.01-1.2; $\mathrm{p}=0.02$; Table 3). No associations were found between the presence of ExDYS and age, sex, QRS duration, presence of LBBB, LVEDV ${ }_{\text {index' }}$ or the presence of rDYS. In the multiple stepwise regression model, ICM remained an important predictor of ExDYS (OR $4.3 ; 95 \% \mathrm{Cl} 1.2-15.7 ; \mathrm{p}=0.03)$. In patients with ICM the presence of ExDYS correlated with a higher $\mathrm{E} / \mathrm{e}^{\prime}$ ratio (OR 1.2; $95 \% \mathrm{Cl} 1.1-1.4 ; \mathrm{p}=0.006)$.

Exercise resulted in a change of rDYS status in 19 (39.5\%) patients (Fig. 1, 2). Fourteen (73.7\%) regained LV synchronicity, whereas five (26.3\%) became dyssynchronous. All five patients who became dyssynchronous at peak exercise had ICM. Among patients who resynchronised at peak exercise, 11 (78.6\%) had DCM and three (21.4\%) had ICM $(p=0.11)$. Exercise-induced resynchronisation was associated with a lower rest $\mathrm{E} / \mathrm{e}^{\prime}$ ratio (OR 0.85; 95\% Cl 0.75-0.97; $\mathrm{p}=0.02)$ and a lower peak exercise $\mathrm{E} / \mathrm{e}^{\prime}$ ratio (OR 0.87; 95\% Cl 0.75-0.99; $\mathrm{p}=0.049$ ) (Table 4). The rest $\mathrm{E} / \mathrm{e}^{\prime}$ ratio remained an important predictor of exercise-induced resynchronisation in the multiple stepwise regression model (OR 0.85; 95\% Cl 0.75-0.97; p = 0.02). No associations were found between exercise-induced dyssynchronisation and its potential predictors. 
Table 3. Associations between the presence of exercise dyssynchrony and its predictors

\begin{tabular}{|c|c|c|c|c|c|}
\hline \multirow{2}{*}{ Variable } & \multicolumn{3}{|c|}{ Univariate analysis } & \multicolumn{2}{|c|}{ Multivariate logistic analysis $\left(R^{2}=0.28\right)$} \\
\hline & OR $[95 \% \mathrm{Cl}]$ & $\mathbf{r}$ & p & OR $[95 \% \mathrm{Cl}]$ & $\mathbf{p}$ \\
\hline Aetiology [0 - non-ischaemic; 1 - ischaemic] & $4[1.2-13.2]$ & 0.14 & 0.02 & $4.3[1.2-15.7]$ & 0.03 \\
\hline LVEF [\%] & $0.88[0.78-0.98]$ & -0.16 & 0.02 & $0.87[0.77-0.98]$ & 0.02 \\
\hline$E / e^{\prime}$ & $1.11[1.01-1.2]$ & 0.17 & 0.02 & & \\
\hline
\end{tabular}

$\mathrm{Cl}$ - confidence interval; OR — odds ratio; other abbreviations — see Table 2

Table 4. Associations between exercise-induced left ventricular resynchronisation and its predictors

\begin{tabular}{lccccccc} 
Variable & \multicolumn{3}{c}{ Univariate analysis } & & & \multicolumn{2}{c}{ Multivariate logistic analysis $\left(\mathbf{R}^{2}=\mathbf{0 . 2 7}\right)$} \\
\cline { 2 - 3 } & OR [95\%Cl] & $\mathbf{r}$ & $\mathbf{p}$ & & OR [95\% Cl] & $\mathbf{p}$ \\
\hline E/e' ratio & $0.85[0.75-0.97]$ & -0.27 & 0.02 & & $0.85[0.75-0.97]$ & 0.02 \\
SE/e' ratio & $0.87[0.75-0.99]$ & -0.18 & 0.049 & & & \\
\hline
\end{tabular}

Abbreviations - see Tables 2 and 3

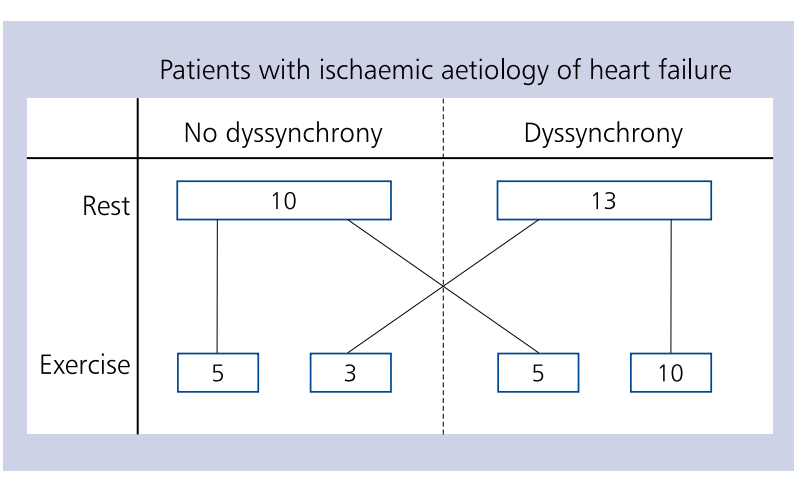

Figure 1. Changes in the number of patients with ischaemic aetiology of heart failure with and without dyssynchrony at rest and at exercise

\section{DISCUSSION}

In the present study we demonstrated that patients with ICM were more prone to have ExDYS as compared to patients with DCM. Patients with ExDYS had poorer systolic and diastolic LV function and more severe MR than patients without ExDYS. We also found that exercise caused changes in rDYS status in some patients. Exercise-induced resynchronisation was more likely to occur in patients with less advanced LV diastolic dysfunction, whereas exercise-induced dyssynchronisation occurred only in patients with ICM.

HFrEF remains one of the most important causes of mortality among cardiovascular diseases, being a real challenge for modern cardiology [6]. Improvement in our understanding of the pathophysiology of the failing heart is essential to bring improve the diagnosis and treatment of HFrEF.

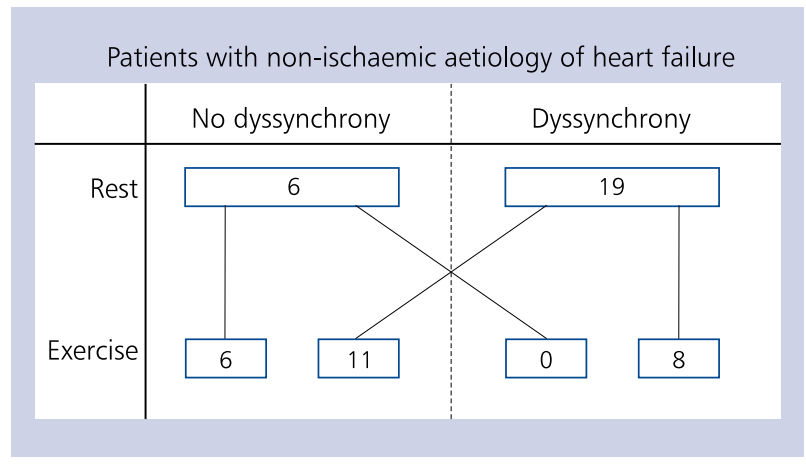

Figure 2. Changes in the number of patients with non-ischaemic aetiology of heart failure with and without dyssynchrony at rest and at exercise

Intraventricular dyssynchrony reflects inhomogeneous timing of contraction of different myocardial segments, caused by disturbed myocyte stimulation or impaired contractility $[7,8]$. In the presence of dyssynchrony, systolic performance of the LV declines at an increased workload, promoting unfavourable cardiac remodelling [9].

Dyssynchrony has been associated with poorer exercise capacity, higher risk of HF decompensation, and death [10-12].

Cardiac resynchronisation therapy has become a standard therapeutic method directed at restoring coordinated ventricular contraction in patients with HFrEF. Despite its positive clinical impact, a significant portion of patients fail to respond sufficiently. Therefore, along with more precise lead positioning [13] and post-implantation device optimisation, 
investigators have been seeking to refine selection criteria of the recipient population [14].

The presence of dyssynchrony has been suggested to improve identification of CRT responders. The weight of current evidence supports the use of echocardiography to detect LV dyssynchrony as a measure of CRT response [15-22].

Studies exploring the pathophysiology of dyssynchrony have indicated that it may be a dynamic phenomenon. In fact, dyssynchrony may persist or change during exercise, under pharmacological stress, or through alteration of the loading conditions. Lafitte et al. [23], who studied the effects of exercise on LV dyssynchrony in a broad spectrum of HFrEF patients, showed that ExDYS was present in up to $69 \%$ of cases. They also found that in up to $26 \%$ of patients either induction or normalisation of ventricular dyssynchrony through exercise may occur. Lancellotti et al. [1] demonstrated that an increase in LV dyssynchrony during exercise was strongly associated with the increase in severity of MR and impairment of LV stroke volume. Similarly, dynamic impairment of intraventricular synchronicity during physical effort was strongly related to exercise incapacity, as demonstrated by D'Andrea et al. [2]. Furthermore, ExDYS has proven to be an important predictor of adverse outcome in patients with HFrEF [24]. On the other hand, the presence of ExDYS has been found to be a better predictor of LV reverse remodelling and functional improvement after CRT than rDYS [3, 25].

Because the majority of previous studies concentrated on the effects of exercise-induced changes of LV dyssynchrony on cardiac performance, prognosis, or CRT response, we attempted to investigate the differences in clinical characteristics between patients with and without ExDYS among subjects with HFrEF and prolonged QRS duration, and to explore potential determinants of the presence of ExDYS.

In our study we found that patients with ExDYS had poorer systolic and diastolic LV function and more severe MR than patients without ExDYS. There were no differences in QRS duration or prevalence of LBBB between the two groups. The prevalence of rDYS was similar. These findings may in part be attributed to the previously reported fact that exercise-induced dyssynchrony worsens LV systolic function and MR, irrespective of QRS duration or morphology [26]. Repeated dyssynchronisation during exercise may augment the impairment of cardiac performance with time. On the other hand, persistence of rDYS during exercise may reflect a more advanced stage of cardiac failure. Therefore, not only exercise-induced dyssynchronisation, as reported previously $[27,28]$, but also persistence of rDYS during exercise may contribute to HF symptom development and reduced functional capacity and might have an impact on prognosis.

We also found that the majority of patients with ExDYS had ICM, while those without ExDYS had DCM. Furthermore, all patients who lost synchronicity during exercise had ICM. Ischaemic aetiology was found to be an important determi- nant of ExDYS. Our data go beyond the findings of Lafitte et al. [23], who showed that $80 \%$ of patients who experienced exercise-induced dyssynchronisation had ICM [23]. We found that patients with ICM are also more prone to remain dyssynchronous during exercise.

The presence of ExDYS and its persistence during exercise in some ICM and DCM patients, as shown in our study, may suggest that part of the myocardium lost its ability to contract. Conversely, dynamic restoration of LV synchronicity during exercise, observed mainly in patients with DCM, could reflect the presence of CR. These findings might suggest a close relationship between ExDYS and myocardial viability. There are, however, conflicting data regarding such a relationship. Several studies have shown that preserved CR helps to unmask LV dyssynchrony, improving the process of CRT patient selection [29-31]. Other authors have demonstrated the opposite. A study by AlJaroudi et al. [32] showed that the presence of stress-inducible ischaemia or the presence of scar on positron emission tomography perfusion images in patients with ICM favours the occurrence of ExDYS. We observed in our study that, because LVEF and WMSI were more severely impaired in patients with ExDYS than in those without, exercise resulted in no change in LVEF and a decrease in WMSI in both groups. The presence of CR was, however, not found to correlate with ExDYS or any change of LV dyssynchrony status either in ICM or DCM patients. This may suggest that ExDYS is not solely dependent on the presence or absence of myocardial viability. As well as systolic properties of the $\mathrm{LV}$, diastolic properties may also have an impact on the dyssynchrony status. The results of our study showed that more advanced diastolic dysfunction of the LV was associated with the presence of ExDYS, especially in ICM patients. Furthermore, exercise-induced resynchronisation was found to be more likely to occur in patients with lower rest and exercise $\mathrm{E} / \mathrm{e}^{\prime}$ ratio. These findings are in line with the data shown by Park et al. [33], who demonstrated that modulation of LV loading conditions affects the dyssynchrony status. They observed a significant decrease in the extent of LV dyssynchrony by reducing the $\mathrm{LV}$ filling pressure and its increase by elevating the afterload. Also, acute changes in the regional wall stress may alter electromechanical coupling within myocardial walls generating dynamic LV dyssynchrony. Increased myocardial stiffness may be a potential explanation of why ICM patients are more prone to experience ExDYS.

ExDYS is an important element of HF pathophysiology. It has been shown to be a promising parameter for selecting CRT candidates. Nonetheless, the mechanisms underlying ExDYS and its dynamic nature have not been entirely elucidated. Whether ExDYS indicates an advanced stage of HF or potentially modifiable comorbidity requires further studies.

The main strength of our study is that it enriches a limited body of literature on the pathomechanisms of exercise-related LV dyssynchrony and helps to improve our understanding of 
this phenomenon. This was a prospective study which evaluated the role of rest and exercise LV dyssynchrony assessed by echocardiography for LV systolic and diastolic functions, according to a predefined protocol.

Despite these advantages there are several limitations. It was a single-centre investigation with a relatively small number of participants. We used a limited number of LV dyssynchrony parameters. We did not evaluate the role of exercise dyssynchrony on the results of CRT. Despite these drawbacks, we believe that the consistency of the results validates the observations. Larger-scale prospective studies are needed to confirm our results.

In conclusion, ischaemic aetiology of HFrEF is an important predictor of ExDYS. Restoration of LV synchronicity during exercise is more likely in patients with less advanced LV diastolic dysfunction.

\section{Conflict of interest: none declared}

\section{References}

1. Lancellotti P, Stainier PY, Lebois F, et al. Effect of dynamic left ventricular dyssynchrony on dynamic mitral regurgitation in patients with heart failure due to coronary artery disease. Am J Cardiol. 2005; 96(9): 1304-1307, doi: 10.1016/j.amjcard.2005.06.077, indexed in Pubmed: 16253603.

2. D'Andrea A, Caso P, Cuomo S, et al. Effect of dynamic myocardial dyssynchrony on mitral regurgitation during supine bicycle exercise stress echocardiography in patients with idiopathic dilated cardiomyopathy and 'narrow' QRS. Eur Heart J. 2007; 28(8): 1004-1011, doi: 10.1093/eurheartj/ehm021, indexed in Pubmed: 17400608.

3. Rocchi G, Bertini M, Biffi M, et al. Exercise stress echocardiography is superior to rest echocardiography in predicting left ventricular reverse remodelling and functional improvement after cardiac resynchronization therapy. Eur Heart J. 2009; 30(1): 89-97, doi: 10.1093/eurheartj/ehn483, indexed in Pubmed: 18987095 .

4. Lang R, Badano L, Mor-Avi V, et al. Recommendations for Cardiac Chamber Quantification by Echocardiography in Adults: An Update from the American Society of Echocardiography and the European Association of Cardiovascular Imaging. Eur Heart J Cardiovasc Imaging. 2015; 16(3): 233-271, doi: 10.1093/ehjci/jev014.

5. McMurray JJV, Adamopoulos S, Anker SD, et al. ESC Guidelines for the diagnosis and treatment of acute and chronic heart failure 2012: The Task Force for the Diagnosis and Treatment of Acute and Chronic Heart Failure 2012 of the European Society of Cardiology. Developed in collaboration with the Heart Failure Association (HFA) of the ESC. Eur Heart J. 2012; 33(14): 1787-1847, doi: 10.1093/eurheartj/ehs104, indexed in Pubmed: 22611136.

6. Pikala M, Maniecka-Bryła I. Fifteen-year mortality trends due to cardiovascular diseases in Poland using standard expected years of life lost, 2000-2014. Kardiol Pol. 2017; 75(10): 1033-1040, doi: 10.5603/KP.a2017.0124, indexed in Pubmed: 28715075.

7. Cheng A, Helm RH, Abraham TP. Pathophysiological mechanisms underlying ventricular dyssynchrony. Europace. 2009; 11 (Suppl 5): v10-v14, doi: 10.1093/europace/eup272, indexed in Pubmed: 19861385.

8. Bleeker GB, Bax JJ, Steendijk P, et al. Left ventricular dyssynchrony in patients with heart failure: pathophysiology, diagnosis and treatment. Nat Clin Pract Cardiovasc Med. 2006; 3(4): 213-219, doi: 10.1038/ncpcardio0505, indexed in Pubmed: 16568130.
9. Kirk JA, Kass DA. Electromechanical dyssynchrony and resynchronization of the failing heart. Circ Res. 2013; 113(6): 765-776, doi: 10.1161/CIRCRESAHA.113.300270, indexed in Pubmed: 23989718.

10. Bajraktari G, Batalli A, Poniku A, et al. Left ventricular markers of global dyssynchrony predict limited exercise capacity in heart failure, but not in patients with preserved ejection fraction. Cardiovasc Ultrasound. 2012; 10(1): 36, doi: 10.1186/1476-712010-36, indexed in Pubmed: 22966942.

11. Bader H, Garrigue $S$, Lafitte $S$, et al. Intra-left ventricular electromechanical asynchrony. A new independent predictor of severe cardiac events in heart failure patients. J Am Coll Cardiol. 2004; 43(2): 248-256, indexed in Pubmed: 14736445.

12. Cho GY, Song JK, Park WJ, et al. Mechanical dyssynchrony assessed by tissue Doppler imaging is a powerful predictor of mortality in congestive heart failure with normal QRS duration. J Am Coll Cardiol. 2005; 46(12): 2237-2243, doi: 10.1016/j. jacc.2004.11.074, indexed in Pubmed: 16360052.

13. Młynarska A, Młynarski R, Gołba KS, et al. Gender-related differences in coronary venous anatomy: a potential basis for various response to cardiac resynchronisation therapy. Kardiol Pol. 2017; 75(3): 247-254, doi: 10.5603/KP.a2016.0153, indexed in Pubmed: 27747855.

14. Gilewski W, Błażejewski J, Karasek D, et al. Are changes in heart rate, observed during dobutamine stress echocardiography, associated with a response to cardiac resynchronisation therapy in patients with severe heart failure? Results of a multicentre ViaCRT study. Kardiol Pol. 2018; 76(3): 611-617, doi: 10.5603/KP.a2017.0261, indexed in Pubmed: 29297189.

15. Marsan NA, Bleeker GB, Ypenburg C, et al. Left ventricular dyssynchrony predicts benefit of cardiac resynchronization therapy in patients with end-stage heart failure before pacemaker implantation. Am J Cardiol. 2003; 92(10): 1238-1240, doi: 10.1016/j. jacc.2004.08.016, indexed in Pubmed: 14609610.

16. Yu CM, Fung WH, Lin H, et al. Predictors of left ventricular reverse remodeling after cardiac resynchronization therapy for heart failure secondary to idiopathic dilated or ischemic cardiomyopathy. Am J Cardiol. 2003; 91(6): 684-688, indexed in Pubmed: 12633798.

17. Nelson GS, Curry CW, Wyman BT, et al. Predictors of systolic augmentation from left ventricular preexcitation in patients with dilated cardiomyopathy and intraventricular conduction delay. Circulation. 2000; 101(23): 2703-2709, indexed in Pubmed: 10851207.

18. Chung ES, Leon AR, Tavazzi L, et al. Results of the Predictors of Response to CRT (PROSPECT) trial. Circulation. 2008; 117(20): 2608-2616, doi: 10.1161/CIRCULATIONAHA.107.743120, indexed in Pubmed: 18458170.

19. Yu CM, Zhang Q, Fung JWH, et al. A novel tool to assess systolic asynchrony and identify responders of cardiac resynchronization therapy by tissue synchronization imaging. J Am Coll Cardiol. 2005; 45(5): 677-684, doi: 10.1016/j.jacc.2004.12.003, indexed in Pubmed: 15734610.

20. Gorcsan J, Tanabe M, Bleeker GB, et al. Combined longitudinal and radial dyssynchrony predicts ventricular response after resynchronization therapy. J Am Coll Cardiol. 2007; 50(15): 1476-1483, doi: 10.1016/j.jacc.2007.06.043, indexed in Pubmed: 17919568.

21. Ruschitzka F, Abraham WT, Singh JP, et al. Cardiac-resynchronization therapy in heart failure with a narrow QRS complex. N Engl J Med. 2013; 369(15): 1395-1405, doi: 10.1056/NEJMoa1306687, indexed in Pubmed: 23998714.

22. Beshai JF, Grimm RA, Nagueh SF, et al. RethinQ Study Investigators. Cardiac-resynchronization therapy in heart failure with narrow QRS complexes. N Engl J Med. 2007; 357(24): 2461-2471, doi: 10.1056/NEJMoa0706695, indexed in Pubmed: 17986493. 
23. Lafitte S, Bordachar P, Lafitte M, et al. Dynamic ventricular dyssynchrony: an exercise-echocardiography study. J Am Coll Cardiol. 2006; 47(11): 2253-2259, doi: 10.1016/j.jacc.2005.11.087, indexed in Pubmed: 16750692.

24. D'Andrea A, Mele D, Nistri S, et al. The prognostic impact of dynamic ventricular dyssynchrony in patients with idiopathic dilated cardiomyopathy and narrow QRS. Eur Heart J Cardiovasc Imaging. 2013; 14(2): 183-189, doi: 10.1093/ehjci/jes154, indexed in Pubmed: 22872513.

25. Wita K, Mizia-Stec K, Płońska-Gościniak E, et al. Low-dose dobutamine stress echo for reverse remodeling prediction after cardiac resynchronization. Adv Med Sci. 2015; 60(2): 294-299, doi: 10.1016/j.advms.2015.04.004, indexed in Pubmed: 26117588.

26. Izumo M, Lancellotti P, Suzuki K, et al. Three-dimensional echocardiographic assessments of exercise-induced changes in left ventricular shape and dyssynchrony in patients with dynamic functional mitral regurgitation. Eur J Echocardiogr. 2009; 10(8): 961-967, doi: 10.1093/ejechocard/jep114, indexed in Pubmed: 19770114.

27. Kang SJ, Lim HS, Choi BJ, et al. The impact of exercise-induced changes in intraventricular dyssynchrony on functional improvement in patients with nonischemic cardiomyopathy. J Am Soc Echocardiogr. 2008; 21(8): 948-953, doi: 10.1016/j. echo.2008.01.009, indexed in Pubmed: 18325737.

28. Chattopadhyay S, Alamgir MF, Nikitin NP, et al. The effect of pharmacological stress on intraventricular dyssynchrony in left ventricular systolic dysfunction. Eur J Heart Fail. 2008;
10(4): 412-420, doi: 10.1016/j.ejheart.2008.02.004, indexed in Pubmed: 18395672.

29. Stankovic I, Aarones M, Smith HJ, et al. Dynamic relationship of left-ventricular dyssynchrony and contractile reserve in patients undergoing cardiac resynchronization therapy. Eur Heart J. 2014; 35(1): 48-55, doi: 10.1093/eurheartj/eht294, indexed in Pubmed: 23918757.

30. Parsai C, Baltabaeva A, Anderson L, et al. Low-dose dobutamine stress echo to quantify the degree of remodelling after cardiac resynchronization therapy. Eur Heart J. 2009; 30(8): 950-958, doi: 10.1093/eurheartj/ehp050, indexed in Pubmed: 19269987.

31. Płońska-Gościniak E, Kasprzak JD, Kukulski T, et al. Role of low-dose dobutamine echocardiography in predicting response to biventricular pacing. Results from the multicenter Viability in Cardiac Resynchronisation Therapy (ViaCRT) study. Pol Arch Med Wewn. 2016; 126(12): 989-994, doi: 10.20452/pamw.3715, indexed in Pubmed: 27958262.

32. AlJaroudi W, Alraies MC, Menon V, et al. Predictors and incremental prognostic value of left ventricular mechanical dyssynchrony response during stress-gated positron emission tomography in patients with ischemic cardiomyopathy. J Nucl Cardiol. 2012; 19(5): 958-969, doi: 10.1007/s12350-012-9592-z, indexed in Pubmed: 22692626.

33. Park HE, Chang SA, Kim HK, et al. Impact of loading condition on the 2D speckle tracking-derived left ventricular dyssynchrony index in nonischemic dilated cardiomyopathy. Circ Cardiovasc Imaging. 2010; 3(3): 272-281, doi: 10.1161/CIRCIMAGING.109.890848, indexed in Pubmed: 20190282.

Cite this article as: Stępniewski J, Kopeć G, Magoń W, et al. Ischaemic aetiology predicts exercise dyssynchrony in patients with heart failure with reduced ejection fraction. Kardiol Pol. 2018; 76(10): 1450-1457, doi: 10.5603/KP.a2018.0148.

\section{WHAT IS NEW?}

Our study provides a novel insight into the pathophysiology of exercise dyssynchrony in patients with heart failure with reduced ejection fraction. We showed that exercise dyssynchrony is closely related to ischaemic aetiology of heart failure, as opposed to non-ischaemic one. We also showed that exercise caused changes in the rest dyssynchrony status in some patients. Exercise-induced resynchronisation was more likely in patients with less advanced left ventricular diastolic dysfunction, whereas exercise-induced dyssynchronisation occurred only in patients with ischaemic aetiology. 